\title{
A Novel Modification of the AOM/DSS Model for Inducing Intestinal Adenomas in Mice
}

\author{
ANASTASIOS ANGELOU ${ }^{1}$, NIKOLAOS ANDREATOS ${ }^{2}$, EFSTATHIOS ANTONIOU ${ }^{1}$, \\ ARGIRO ZACHARIOUDAKI ${ }^{3}$, GEORGE THEODOROPOULOS ${ }^{4}$, CHRISTOS DAMASKOS $^{1}$, \\ NIKOLAOS GARMPIS ${ }^{1}$, CHUNHUI YUAN ${ }^{2,5}$, WEIDONG XIAO ${ }^{2,6}$, STAMATIOS THEOCHARIS ${ }^{7}$, \\ GEORGE ZOGRAFOS $^{4}$, APOSTOLOS PAPALOIS ${ }^{3}$ and GEORGIOS ANTONIOS MARGONIS ${ }^{2}$ \\ ${ }^{1}$ Second Department of Propedeutic Surgery, Laiko General Hospital, \\ Medical School, National and Kapodistrian University of Athens, Athens, Greece; \\ ${ }^{2}$ Department of Surgery, Johns Hopkins University School of Medicine, Baltimore, MD, U.S.A.; \\ ${ }^{3}$ Experimental Research Centre ELPEN Pharmaceuticals, S.A. Inc., Co., Pikermi, Greece; \\ ${ }^{4}$ First Propaedeutic Department of Surgery, Hippocration Hospital, \\ School of Medicine, National and Kapodistrian University of Athens, Athens, Greece; \\ ${ }^{5}$ Department of General Surgery, Peking University Third Hospital, Beijing, P.R. China; \\ ${ }^{6}$ Department of General Surgery, The First Affiliated Hospital of Nanchang University, Nanchang, P.R. China; \\ ${ }^{7}$ First Department of Pathology, National and Kapodistrian University of Athens, Athens, Greece
}

\begin{abstract}
Background/Aim: Our aim was to develop an animal model of the precancerous stages of colitis-associated carcinogenesis by modifying the established azoxymethanel dextran sulfate sodium (AOM/DSS) protocol. Materials and Methods: Six mice were treated with varying cycles of DSS following AOM administration as above (group 1: three mice received three 5-day cycles of 3.0\% DSS and group 2: three mice received three 7-day cycles of $2.5 \%$ DSS; every cycle was followed by a 2-week rest period) and were sacrificed on day 84 of the experiment. By contrast, three female C57BL6 mice (group 3) were treated with a single intraperitoneal dose (10 mg/kg of body weight) of AOM followed by three 5-day cycles of oral $2.5 \%$ DSS, with each cycle interrupted by a 2week rest period. The mice of this group were sacrificed at 60 days. Results: In groups 1 and 2, cancer was noted in five out of the six mice. In group 3, adenomas with dysplastic lesions were noted in all of the mice, but none had developed adenocarcinoma. Conclusion: Our results suggest that the administration of three 5-day cycles of 2.5\% DSS following an initial dose of AOM may successfully induce adenoma formation without the concurrent presence of carcinoma in
\end{abstract}

Correspondence to: Georgios Antonios Margonis, MD, Ph.D., Johns Hopkins University Medical School, Department of Surgery, Division of Hepatobiliary and Pancreatic Surgery and Division of Surgical Oncology, 600 N. Wolfe Street, Carnegie 137, Baltimore, MD 21287, U.S.A.E-mail: antonis.margonis@gmail.com

Key Words: AOM/DSS, IBD, animal model, adenomas. female C57BL6 mice that are sacrificed on experimental day 60. In turn, this modification of the widely used AOM/DSS protocol may constitute a novel approach for investigating colitis-related colonic adenomas.

Individuals with inflammatory bowel disease, such as Crohn's disease or ulcerative colitis are at risk of developing colitisassociated colorectal cancer (CAC), in proportion to the duration and extent of their disease $(1,2)$. CAC development is known to be a multi-step process, with a median duration of 8 20 years from the inciting molecular event (s) to presentation of clinical disease (3). Specifically, it has been demonstrated that the initial pre-neoplastic changes involve normal epithelial cells that form aberrant crypt foci (ACF). In turn, ACF proliferate by crypt fission to form microadenomas. The resulting microadenomas continue to increase in size, until the accumulation of additional genetic aberrations gives rise to macroscopic adenomas, adenomatous polyps, carcinoma in situ and, ultimately, invasive cancer (4). As such, experimental models that mimic the precancerous stage of adenoma formation shed light on disease progression and may potentially allow for the development of effective chemoprevention $(5,6)$. Both in vitro and in vivo models may be employed for this purpose; however, in vitro models are hampered by their lack of tissue context, an important disadvantage given the central role of the tumor microenvironment in neoplastic proliferation (7). Consequently, in vivo models may provide superior insight into adenoma formation and development.

Dextran sulfate sodium (DSS), that can be administered to mice in multiple cycles via drinking water, induces a chronic 
inflammatory state and has been used extensively in in vivo models of CAC development (8). Importantly, it has been observed that the use of DSS following prior administration of the mutagen azoxymethane (AOM) speeds up the process of tumor development from several months to as little as 810 weeks, leading to the widespread acceptance of the AOM/DSS model for CAC (9). The same basic approach could theoretically be adapted in such a way as to induce precancerous lesions rather than invasive cancer; however, to our knowledge, an AOM/DSS protocol that reliably induces colitis-associated adenoma formation in mice has yet to be described. We aimed to develop such a protocol through appropriate modification of the widely employed AOM/DSS model of CAC development.

\section{Materials and Methods}

Experimental conditions, animals and employed chemicals. Female C57BL6 mice (age: 8 weeks) were used in this study as other investigators have previously recommended (10). They were maintained at the ELPEN Animal Facility, Pikermi, Greece, in compliance with Institutional Animal Care Guidelines and IRB regulations. For the duration of the experiment, all animals were housed in plastic cages (three mice/cage) with free access to drinking water and a pelleted basal diet, under controlled conditions of humidity $(50 \pm 10 \%)$, light $(12 / 12 \mathrm{~h}$ light/ dark cycle) and temperature $\left(23 \pm 2^{\circ} \mathrm{C}\right)$. The experimental animals were quarantined for the first 7 days, and then randomized into experimental and control groups. The colonic carcinogen AOM was purchased from Sigma Chemical Co. (St. Louis, MO, USA), while DSS was purchased from ICN Biochemicals, Inc (Aurora, OH, USA).

Experimental procedure. A total of 12 mice were equally divided $(\mathrm{n}=3)$ into four groups (three experimental groups and one control group). Specifically, group 1 was initially treated with a single intraperitoneal injection of AOM (10 mg/kg of body weight). Starting 1 week after AOM administration, the animals received 3\% DSS in the drinking water for 5 days, followed by a 2-week rest period without DSS, then another 5-day cycle of 3\% DSS followed by a second 2-week rest period and, subsequently, a final 5-day cycle of 3\% DSS. Group 2 was also initially treated with a single intraperitoneal injection of AOM (10 $\mathrm{mg} / \mathrm{kg}$ of body weight). Starting 1 week after AOM administration, the animals received $2.5 \%$ DSS in the drinking water for 7 days, followed by a 2-week rest period without DSS, then another 7-day cycle of $2.5 \%$ DSS followed by a second 2-week rest period and, subsequently, a final 7 -day cycle of $2.5 \%$ DSS. The mice assigned to groups 1 and 2 were all sacrificed at 84 days.

Group 3 was also initially treated with a single intraperitoneal injection of AOM (10 mg/kg of body weight). Starting 1 week after AOM administration, the animals received $2.5 \%$ DSS in the drinking water for 5 days, followed by a 2 -week rest period without DSS, then another 5-day cycle of $2.5 \%$ DSS followed by a second 2-week rest period and, subsequently, a final 5-day cycle of $2.5 \%$ DSS. The mice of the third group were sacrificed at 60 days. Group 4 (no AOM) served as a control group and all the mice assigned to it were sacrificed at 84 days.

Following sacrifice of the experimental animals, the colon was flushed with saline and excised in each case. Colon length from the ileocecal junction to the anal verge was measured and the colon was cut open longitudinally along its main axis and washed with saline. Subsequently, the colon was macroscopically inspected, sectioned, and fixed in $10 \%$ buffered formalin for a minimum of 24 hours. The resulting paraffin-embedded sections underwent hematoxylin \& eosin (H\&E) staining and were examined for the presence of histological alterations, such as mucosal ulceration, dysplasia, ACF, and carcinoma.

\section{Results}

General findings. All mice developed loose stools (without gross evidence of blood) during DSS administration and experienced a mean weight reduction of $10 \%$. All mice recovered clinically during the water-only rest period. Following sacrifice of the mice assigned to the three experimental groups, histological examination revealed colitis of varying severity. Characteristic findings included mucosal ulceration, infiltration with mononuclear and polymorphonuclear leukocytes in the lamina propria and submucosa, hyperplastic epithelia and ulcers in the process of healing via re-epithelialization.

Comparison of histological lesions. Group 1: Two out of three mice developed adenocarcinoma in multiple sites of the colon. A single mouse did not develop adenocarcinoma, but exhibited multiple sites of hyperplasia and multiple colonic adenomas on histologic examination. The examined lesions were found to be compatible with dysplastic ACF and microadenoma, respectively.

Group 2: All mice developed adenocarcinoma of the colon, as well as multiple adenomas and evidence of hyperplasia.

Group 3: All mice developed sites of colonic hyperplasia, with one or more crypts per microscopic field characterized by a thickened layer of epithelial cells (that stained more intensely with methylene blue), a slit-shaped luminal opening, increased pericryptal space and apparent elevation. While adenomas with dysplastic epithelium were occasionally noted, the presence of adenocarcinoma was not demonstrated in any of the examined sections. Benign, circumscribed neoplasm composed of tubular and/or villous structures lined by dysplastic epithelium were developed as well.

Group 4: No abnormalities were noted during histopathological examination.

\section{Discussion}

In vivo models of colonic carcinogenesis (especially those using the AOM/DSS protocol) are effective means of studying the early, precancerous stages of CAC, due to their capacity to closely mimic the normal epithelium-ACFadenoma-carcinoma sequence that takes place in the human colon (11). Importantly, such models are also ideal for 
testing the efficacy of chemopreventive compounds that aim to arrest the development of cancer, as demonstrated by a recent meta-analysis (12). However, while reliable and relatively inexpensive in vivo models of carcinogenesis have been developed, the possibility of modeling adenoma development with the aid of the AOM/DSS protocol has received far less attention. For example, while DeRobertis et al. and Ward reported that AOM/DSS-induced tumors typically display adenomatous growth before the development of malignancy, only very limited attempts to determine the precise experimental protocols (timing of mouse sacrifice, AOM/DSS dose, mouse breed etc.) which are needed to promote the development of adenomas rather than carcinomas have been made to date $(13,14)$.

In fact, only a single study by Suzuki et al., to our knowledge, described a protocol that promotes the development of adenomas in mice (11). Specifically, the authors reported that after male ICR mice were treated with a single intraperitoneal injection of AOM $(10 \mathrm{mg} / \mathrm{kg}$ of body weight) and $2 \%(\mathrm{w} / \mathrm{v})$ DSS (for 7 days) 1 week after the AOM injection, colonic adenomas developed in two out of five mice ( $40 \%$ incidence and $0.40 \pm 0.49$ multiplicity) in week 3 and colon cancer developed in two out of five (40\% incidence and 2.00 \pm 3.52 multiplicity) mice in week 4 (11). Unsurprisingly, the incidence of carcinoma gradually increased with time, reaching $100 \%$ (6.20 \pm 2.48 multiplicity) in week 6 . While their protocol suggests the means to promote adenoma formation in mice, its applicability is unfortunately limited. Firstly, the ICR breed of mice is not commonly used today. In turn, this is a very serious limitation as more commonly used breeds of mice demonstrate an extremely variable propensity to develop cancer following AOM/DSS exposure (Balb/c>C57BL/6N $>\mathrm{C} 3 \mathrm{H} / \mathrm{HeN}=\mathrm{DBA} / 2 \mathrm{~N})$ (15). As such, an AOM/DSS protocol developed in a given mouse breed is likely not directly applicable to other breeds, considerably weakening the utility of the findings reported by Suzuki et al. Moreover, even if the aforementioned protocol could be directly applied across different mouse breeds the relatively low success rate and close temporal relationship of adenoma and carcinoma development pose serious practical difficulties and suggest that an alternative approach should be explored.

In contrast, we were able to develop a potentially more efficient (all three animals in group 3 developed adenomas), experimental model of colonic adenoma development in female mice of the commonly employed C57/BL6 breed. The protocol was based on a single intraperitoneal injection of AOM $(10 \mathrm{mg} / \mathrm{kg}$ of body weight) followed by three cycles of $2.5 \%$ DSS and sacrifice of the experimental animals on day 60 . Importantly, at sacrifice, all group 3 animals had developed abnormally large, darkly staining and slightly raised aberrant crypts as well as adenomas without the presence of adenocarcinomas. While group numbers were too small to allow for more definitive conclusions, it appears that these results may largely stem from the earlier sacrifice time point; indeed, group 2 mice that received comparable doses of AOM/DSS had all developed cancer as well as adenomas and hyperplasia by day 84 . This suggests that the group 3 protocol accurately replicated the normal epithelium-ACF-adenoma-carcinoma sequence, and given more time would likely have resulted in the development of cancer.

The principal limitation of this study was the relatively small sample size, which, however, is a common issue in pilot studies of this nature across the literature. Nonetheless, replication of our results in a larger number of experimental animals will provide a better estimate of the protocol's true efficacy in promoting the development of adenoma rather than carcinoma. Moreover, as discussed above, our findings were based on female mice of the C57/BL6 breed and cannot be extended to other mouse breeds without prior validation. While the C57/BL6 breed is commonly employed, inexpensive and readily available, it remains possible that the needs of future experiments will necessitate the use of other breeds; in such cases, caution should be exercised before attempting to replicate our findings.

In conclusion, the AOM/DSS animal model was shown to be a powerful tool for investigating the pathogenesis of CAC. In the present study, we described a modification of this widely employed protocol that might allow for colitisassociated adenoma growth to be modeled, before the development of carcinoma ensues. In turn, capturing disease in that early stage might facilitate the detection of possible tumor-risk modifiers, as well as enabling the testing of novel chemopreventive agents that aim to arrest the development of CAC. Future studies are needed to validate our findings.

\section{Conflicts of Interest}

None to report.

\section{Acknowledgements}

The current experimental study was supported as research scholarship by the experimental-research center ELPEN.

\section{References}

1 Grivennikov SI: Inflammation and colorectal cancer: colitisassociated neoplasia. Semin Immunopathol 35: 229-244, 2013.

2 Neumann H, Vieth M, Langner C, Neurath MF and Mudter J: Cancer risk in IBD: how to diagnose and how to manage DALM and ALM: World J Gastroenterol 17: 3184-3191, 2011.

3 Shanahan F: Review article: colitis-associated cancer - time for new strategies. Aliment Pharmacol Ther 18(Suppl 2): 6-9, 2003.

4 Kinzler KW and Vogelstein B: Lessons from hereditary colorectal cancer. Cell 87: 159-170, 1996. 
5 Tanaka T: Preclinical cancer chemoprevention studies using animal model of inflammation-associated colorectal carcinogenesis. Cancers (Basel) 4: 673-700, 2012.

6 Tanaka T, Kohno H and Mori H: Chemoprevention of Colon Carcinogenesis by Dietary Non-nutritive Compounds. Asian Pac J Cancer Prev 2: 165-177, 2001.

7 Sharpless NE and Depinho RA: The mighty mouse: genetically engineered mouse models in cancer drug development. Nat Rev Drug Discov 5: 741-754, 2006.

8 Neufert C, Becker C and Neurath MF: An inducible mouse model of colon carcinogenesis for the analysis of sporadic and inflammation-driven tumor progression. Nat Protoc 2: 19982004, 2007.

9 Tanaka T, Kohno H, Suzuki R, Yamada Y, Sugie S and Mori H: A novel inflammation-related mouse colon carcinogenesis model induced by azoxymethane and dextran sodium sulfate. Cancer Sci 94: 965-973, 2003.

10 Thaker AI, Shaker A, Rao MS and Ciorba MA: Modeling colitisassociated cancer with azoxymethane (AOM) and dextran sulfate sodium (DSS). J Vis Exp, 2012. doi: 10.3791/4100

11 Suzuki R, Kohno H, Sugie S and Tanaka T: Sequential observations on the occurrence of preneoplastic and neoplastic lesions in mouse colon treated with azoxymethane and dextran sodium sulfate. Cancer Sci 95: 721-727, 2004.
12 Corpet DE and Pierre F: How good are rodent models of carcinogenesis in predicting efficacy in humans? A systematic review and meta-analysis of colon chemoprevention in rats, mice and men. Eur J Cancer 41: 1911-1922, 2005.

13 De Robertis M, Massi E, Poeta ML, Carotti S, Morini S, Cecchetelli L, Signori E and Fazio VM: The AOM/DSS murine model for the study of colon carcinogenesis: From pathways to diagnosis and therapy studies. J Carcinog 10: 9, 2011.

14 Ward JM: Morphogenesis of chemically induced neoplasms of the colon and small intestine in rats. Lab Invest 30: 505-513, 1974.

15 Suzuki R, Kohno H, Sugie S, Nakagama H and Tanaka T: Strain differences in the susceptibility to azoxymethane and dextran sodium sulfate-induced colon carcinogenesis in mice. Carcinogenesis 27: 162-169, 2006.
Received April 2, 2018

Revised May 4, 2018

Accepted May 9, 2018 